the statement of the authors may be noted that in their 39 cases a rash was present in 22 .

The symptoms due to the inflammation of the meninges, viz., severe headache, vomiting, retraction of the head and neck, stiffness of the neck, and the presence of Kernig's sign, are common to all forms of meningitis. But the presence of the rash, and the discovery of the Meningococcus (diplococcus) intracellularis in the cerebro-spinal fluid after withdrawal by lumbar puncture, constitute the essential differential diagnostic signs. Excellent photographs are given illustrating cases exhibiting the head retraction and Kernig's sign; also remarkably well-executed coloured plates illustrating the macroscopic appearances presented by the brain and spinal cord, and the microscopic appearances of the meninges and the cerebro-spinal fluid containing the diplococcus intracellularis.

Various statistics are quoted which appear to prove that the authors are right in asserting that frequent lumbar puncture is the most valuable therapeutic measure, and that it is not enhanced by subsequent intrathecal injection of Flexner's serum. In fact, they state: "In our somewhat limited experience the introduction of serum caused, for the most part, a decided aggravation of cerebral symptoms." An excellent chapter on the pathology of the disease follows, in which the authors discuss the channels by which the diplococcus passes from the nasopharynx to the subarachnoid space. This is followed by a chapter on changes in the cerebrospinal fluid and the cultivation of the meningococcus from it, from the blood, and from the urine. The last fifty pages are devoted to an exhaustive account of the epidemiology and bacteriology, in which are discussed the contagion direct from throat to throat, the mode of examination of carriers, and their treatment by isolation and local applications to the throat and nose. In conclusion, there is an appendix containing a remarkable example of the spread of the meningococcus from carrier to carrier.

\section{A MONOGRAPH ON TICKS.}

Ticks. A Monograph of the Ixodoidea. Part iii. The Genus Haemaphysalis. By Prof. G. H. F. Nuttall and C. Warburton. October, I9 5. Pp. xiii + 349-550 + plates viii-xiii. (Cambridge: At the University Press.) Price I2s. net.

Bibliography of the Ixodoidea. Part ii. May, I9I 5. By Prof. G. H. F. Nuttall and L. E. Robinson. Pp. 32. (Cambridge: At the University Press.) Price $4 s$. 6d. net.

THE present part of this useful monograph deals with the fifty species and varieties of Hæmaphysalis recognised as valid by the authors. The distinguishing features of the genus are stated and discussed, and the difficulty is noted of finding, among the many negative characters NO. 2438 , VOL. 97$]$ in this genus, points which can be employed for differentiating the species. Nevertheless, the authors have succeeded in drawing up a helpful dichotomic key for the determination of the species. The species are then considered in turn, and, as in the two previous parts of the monograph, careful drawings are given of those parts which are of systematic importance. Interesting conclusions are reached from a study of the geographical distribution of the different species; e.g., that $H$. bispinosa has almost certainly been imported into East Africa, and possibly into New South Wales, with Indian cattle. Only one species of Haemaphysalis appears to be restricted to birds, whereas several species of Ixodes are found only on birds. The authors give a list of hosts on which the various species of Hæmaphysalis have been found, and discuss the condemned and doubtful species. An account is given of all that is known regarding the biology of six species, two of which have been proved to be the carriers of pathogenic protozoa, one especially-H. leachi-being known in many parts of Africa as the carrier of a fatal disease-canine piroplasmosis or malignant jaundice.

The bibliography ( 462 titles) contains references to, and in many cases short notes on the nature and contents of, papers which for the most part have appeared since the publication of the previous bibliography in $19 \mathrm{I} I$.

\section{OUR BOOKSHELF.}

Newsholme's School Hygiene. The Laws of Health in relation to School Life. New edition, rewritten for all School Workers, by Dr. J. Kerr. Pp. 352. (London: G. Allen and Unwin, Ltd., n.d.) Price $4 s$. $6 d$. net.

Newsholme's text-book on school hygiene first appeared in 1887 , and in 1912 it reached its thirteenth edition. That fact is sufficient evidence of the appreciation it has met with; but circumstances have not made it possible for Dr. Newsholme to continue to develop the work so as to keep it abreast of the rapid advance of the science of school hygiene and the extension of its practice which recent years have witnessed. Hence it became desirable that the text-book should be rewritten by one who, like Dr. James Kerr, has played a more prominent part in these developments. The result is a text-book possessing much merit, and embodying facts and opinions based upon a large amount of experience and research.

It seems from a perusal of the first paragraph that the book is more particularly designed for school-teachers, but to such it will be more satisfactory when Dr. Kerr is able in the next edition to bring his exceptional knowledge and experience to bear upon a fuller treatment of some matters of importance; for while the book is (generally speaking) well balanced in its treatment of the subject-matter, it is in places much too brief. To 